\title{
Midnight Salivary Cortisol and Other Effective Factors in the Graduation of Clinical Suspect of Cushing Syndrome: Is There Any Reasonable Clinical Score?
}

\author{
Meral Mert ${ }^{1}$, Refik Tanakol ${ }^{1}$, Hande Karpuzoglu ${ }^{2}$, Semra Abbasoglu², Sema Yarman ${ }^{1}$, \\ Harika Boztepe ${ }^{1}$, Faruk Alagol ${ }^{1}$ \\ ${ }^{1}$ Department of Internal Medicine, Division of Endocrinology and Metabolism, Istanbul Medical Faculty, Istanbul University, \\ Istanbul, Turkey \\ ${ }^{2}$ Department of Biochemistry, Istanbul Medical Faculty, Istanbul University, Istanbul, Turkey \\ Email: meralmert@hotmail.com
}

Received October 14, 2012; revised November 2, 2012; accepted December 22, 2012

\begin{abstract}
Background: Diagnosis of Cushing's Syndrome (CS) at the right time and with the right method is getting more important for the patients and clinicians due to high mortality rate. The most appropriate laboratory test will provide great benefits in terms of cost-effectiveness in the well-chosen group of patients. Selection of the high risk group is of crucial importance for the true diagnosis and treatment on time. Aim: The aim of this study was to evaluate the worth of the midnight salivary cortisol and to establish other effective factors in the graduation of clinical suspect of CS. Material and Methods: 115 patients were evaluated in weight, height, body mass index (BMI), waist/hip ratio, systolic, diastolic blood pressures, hirsutism, weight gain, purple-stria, plethore, buffalo-hump, supraclavicular fullness, temporal fat cushion, acnea, moonface, proximal muscle weakness, lower limb edema, ecchymosis, loss of libido, depression, diabetes mellitus, hypertension, allopecia of all patients were noted in the evaluation forms (23 findings). Patients were grouped according to clinical scores, low $(<8)$, medium $(8-16)$ and high $(>16)$. Results: When we compare the groups in terms of midnight salivary cortisol, morning salivary cortisol after overnight dexamethasone suppression test, we found statistically significant relationship between the low and high clinical score groups, as well as between medium and high score groups (p: 0.0001). Urinary free cortisol was statistically significant only between low and high clinical score groups (p: 0.0001). Conclusion: This clinical scoring system which includes clinical signs and laboratory findings both, can be used for selection of the high risk group.
\end{abstract}

Keywords: Salivary Cortsiol; Clinical Suspect; Clinical Score; Cushing’s Syndrome

\section{Introduction}

Cushing's syndrome (CS) develops as a result of increased exposure to glucocorticoids and if untreated, mortality can reach up to $50 \%$ within five years in this serious disease [1]. Incidence rates range from 2 to 5 in a million, pegged in the epidemiological studies from different countries $[2,3]$. These rates were found to be higher in smaller sub-group analysis, such as obesity, adrenal incidentaloma and uncontrolled type 2 diabetes mellitus groups. Therefore, the diagnosis of CS at the right time and with the right method is getting more important for the patients and clinicians.

Weight gain with redistribution of adipose mass centrally, dorsocervical fat accumulation, especially supraclavicular and temporal fossa fullness, thinning of the skin with easy bruising, abdominal striae, poor wound healing, immune suppression, rib fractures, hirsutism in women, acne, and muscle wasting leading to proximal muscle weakness and mood changes are common complaints in Cushing's syndrome [4].

Cushing's syndrome despite the lack of specific symptoms of this disease cluster is the emergence of a very common condition. In addition, all patients do not carry all the characteristic findings of the syndrome. Therefore, investigation for the values of the laboratory tests which can be used for the diagnosis and etiology of $\mathrm{CS}$ are important. The measurement of late-night salivary cortisol provides the most sensitive method for screening, and urine-free cortisol and low-dose dexamethasone suppression testing (DST) may be used for confirmation of the diagnosis of endogenous hypercortisolism [5]. The salivary cortisol is a very valuable indicator of the plasma free cortisol. Cortisol is bound to the proteins in the blood. CBG or transcortin is the protein to which $90 \%$ of the cortisol binds while $7 \%$ of the cortisol is 
bound to albumin. The plasma free cortisol is released from the salivary glands and no binding proteins exist in the saliva. It's not affected by the saliva release rate. Saliva is easy to collect and represents a non-invasive method [4].

The most appropriate laboratory test will provide great benefits in terms of cost-effectiveness in the well-chosen group of patients. Selection of the high risk group is of crucial importance for the true diagnosis and treatment on time.

The aim of this study was to evaluate the worth of the midnight salivary cortisol and to establish other effective factors in the graduation of clinical suspect of CS.

\section{Material and Methods}

After receiving the informed concent, 115 patients who has suspicion of Cushing's syndrome (16 male, mean age $35.86 \pm 12.62$ years and 99 female, mean age $38.38 \pm$ 13.93 years) were evaluated in our study. The symptoms, detailed physical findings, weight and height, body mass index (BMI), waist/hip ratio, systolic and diastolic blood pressures and also hirsutism, weight gain, purple-stria, plethore, buffalo-hump, supraclavicular fullness, temporal fat cushion, acnea, moonface, proximal muscle weakness, lower limb edema, ecchymosis, loss of libido, depression, diabetes mellitus, hypertension, allopecia of all patients were noted in the evaluation forms (23 findings). The patients with only 8 findings were accepted to have low clinical score, those with 8 to 16 and 16 to 23 findings were accepted to have clinically moderate and high scores, respectively. Salivary samples were collected from the individuals after 21 p.m. at rest, without using any steroid drops, oinments or medications or without teeth brushing. Salivary samples are collected at midnight and also after having $1 \mathrm{mg}$ dexamethasone tablet orally at $8 \mathrm{am}$ before breakfast. Prior to the test, 24-hour urine samples were collected to detect urinary free cortisol levels. The measurements of patients with adrenal tumors were done after dexamethasone supression tests and other hormon secretion tests.

The salivary, serum and urinary cortisol were measured by Cortisol-LIA 96, Cortisol-ELISA 96 and RIA tests, respectively. SPSS 12 programme was used for statistical analysis. Comparison of the results of the patients was made with One-Way Anova. Local ethic comittee approval was taken.

\section{Results}

Patients with low-medium and high clinical score group had a mean age and mean BMI were found 40.59 \pm 13.16 years, $28.62 \pm 7.9 \mathrm{~kg} / \mathrm{m}^{2}, 34.64 \pm 14.06$ years $30.43 \pm$ $8.38 \mathrm{~kg} / \mathrm{m}^{2}, 40.13 \pm 15.33$ years and $28.77 \pm 7.02 \mathrm{~kg} / \mathrm{m}^{2}$ respectively (Table $\mathbf{1}$ ).

Demographic features of patients were shown in Table 2.

Midnight salivary cortisol and morning salivary cortisol levels after overnight dexamethasone suppression test were higher in the patients with high clinical score. (Figure 1).

As expected in the group with high clinical score, 24 hour urine free cortisol, basal cortisol and blood cortisol levels after $1 \mathrm{mg}$ DST were found in the highest value.

In our study, patients were grouped according to clinical scores, low $(<6)$, medium $(6-12)$ and high $(>12)$. When we compare the groups in terms of midnight salivary cortisol levels, we found statistically significant relationship between the low and high clinical score

Table 1. Serum, urinary and salivary cortisol levels were in three groups.

\begin{tabular}{lccc}
\hline & $\begin{array}{c}\text { Low clinical score (S < 6) } \\
\text { (group 0) (n: 56) }\end{array}$ & $\begin{array}{c}\text { Medium clinical score (S: 6 - 12) } \\
\text { (group 1) (n: 38) }\end{array}$ & $\begin{array}{c}\text { High clinical score(S > 12) } \\
\text { (group 2) (n: 21) }\end{array}$ \\
\hline Midnight salivary cortisol $\mu \mathrm{g} / \mathrm{dl}^{\mathrm{a} 1}$ & $0.20 \pm 0.35$ & $0.148 \pm 0.20$ & $0.880 \pm 1.14$ \\
Morning salivary cortisol after overnight & $0.068 \pm 0.11$ & $0.083 \pm 0.16$ & $0.288 \pm 0.38$ \\
DST $(\mu \mathrm{g} / \mathrm{dl})^{22}$ & $0.88 \pm 0.9$ & $0.84 \pm 0.7$ & $1.45 \pm 1.17$ \\
Serum cortisol after overnight DST $(\mu \mathrm{g} / \mathrm{dl})$ & $36.53 \pm 10.35$ & $28.36 \pm 24.22$ & $77.62 \pm 8.41$ \\
Urinary cortisol $(\mu \mathrm{g} / 24$ hour) & $\mathrm{a3}$ & $16.08 \pm 4.37$ & $20.86 \pm 6.88$ \\
Basal cortisol $(\mu \mathrm{g} / \mathrm{dl})^{\mathrm{a} 4}$ & $15.35 \pm 4.1$ & & \\
\hline
\end{tabular}

One-Way Anova was significant at the p levels: a1: 0 - 2 between groups p: $0.0001,1-2$ in the p: 0.0001 . a2: 0 - 2 between groups p: 0.0001 , $1-2$ in the p: 0.005. a3: 0 - 2 between groups p: 0.0001. a4: 0 - 2 between groups p: $0.0001,1-2$ in the p: $0.002 .{ }^{* *}$ DST: Dexamethasone suppresion test.

Table 2. Demografic features of the patients.

\begin{tabular}{cccc}
\hline & Low clinical score & Medium clinical score & High clinical score \\
\hline Age (year) & $40.59 \pm 13.16$ & $34.64 \pm 14.06$ & $40.13 \pm 15.33$ \\
BMI kg/m & $28.62 \pm 7.9$ & $30.43 \pm 8.38$ & $28.77 \pm 7.02$ \\
\hline
\end{tabular}




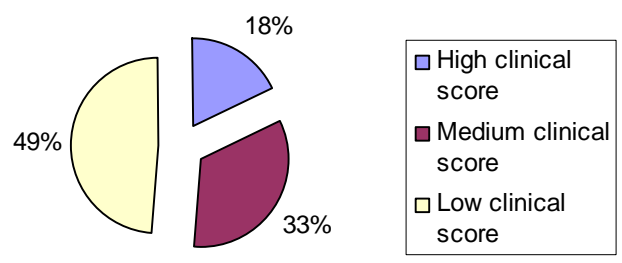

Figure 1. Number of patients according to the clinical scores.

groups, as well as between medium and high score groups (p: 0.0001). Similar relationship was found at morning salivary cortisol after overnight dexamethasone suppression test.

Urinary free cortisol was statistically significant only between low and high clinical score groups (p: 0.0001). Midnight salivary cortisol and morning salivary cortisol after overnight dexamethasone suppression test was found to be statistically significantly higher in high clinical score group.

\section{Discussion}

Cushing's syndrome remains as one of the most challenging endocrine pathologies. The recommended screening tests are 24-h urinary free cortisol, 1-mg overnight dexamethasone suppression test, and late-night salivary cortisol. Clinically identifying high-and low-risk patients may avoid unnecessary loss of time and money. Factors affecting the sensitivity of the tests may be distinctive in different patient groups.

Wide variety of clinical signs suggestive of Cushing's syndrome can be found in patients. Some patients may have atypical findings. Moreover, cyclic Cushing's syndrome, a rare aberrant receptor mediated-CS should be kept in mind for differential diagnosis. The decision of which test will be performed in different risk groups becomes more important.

For this purpose, patients were divided into three groups according to number of signs and symptoms suggesting CS. Serum and salivary cortisol and salivary cortisol after 1 $\mathrm{mg}$ DST were investigated as screening test in these three groups. When we compare the groups in terms of midnight salivary cortisol, we found statistically significant relationship between the low and high clinical score groups, as well as between medium and high score groups (p: 0.0001). Similar relationship was found at morning salivary cortisol after overnight dexamethasone suppression test.

Sanino et al found that clinical severity index (CSI) is a valid and reliable clinimetric method to evaluate severity in CS. Eight clinical features were selected and each one was graded on an ordinal 3-point scale $(0-2)$ with specification of anchor points based on severity in CSI index. Unlike our study, CSI index were evaluated with already diagnosed patients [6].

Ross and Lynch were evaluated discriminatory values of symptoms and signs of Cushing's syndrome in $70 \mathrm{pa}-$ tients who had histological confirmation of the diagnosis. In this study, presence of bruising, muscle weakness, and hypertension are the most discriminating features of the disease that can be detected at the initial examination. Unlike our study, symptoms and signs were evaluated for the discriminatory values of the diagnosis, but we tried to determine a clinical score in order to show who needs much more than screening tests $[7,8]$.

The most appropriate laboratory test will provide great benefits in terms of cost-effectiveness and in the wellchosen group of patients. Selection of the high risk group person is of crucial importance for the true diagnosis and treatment on time. This clinical scoring system which includes clinical signs and laboratory findings both, can be used for selection of the high risk group.

\section{Acknowledgements}

The authors thank Funda Sezgin for statistics.

\section{REFERENCES}

[1] C. M. Plotz, A. I. Knowlton and C. Ragan, "The Natural History of Cushing's Syndrome," The American Journal of Medicine, Vol. 13, No. 5, 1952, pp. 597-614. doi:10.1016/0002-9343(52)90027-2

[2] J. Lindholm , S. Juul , J. O. Jørgensen, et al., "Incidence and Late Prognosis of Cushing's Syndrome: A PopulationBased Study," The Journal of Clinical Endocrinology \& Metabolism, Vol. 86, No.1, 2001, pp. 117-123.

[3] G. Leibowitz, A. Tsur and S. D. Chayen, "Preclinical Cushing's Syndrome: An Unexpected Frequent Cause of Poor Glycemic Control in Obese Diabetic Patients," Clinical Endocrinology, Vol. 44, No. 6, 1996, pp. 717-722. doi:10.1046/j.1365-2265.1996.737558.x

[4] James W. Findling and Hershel Raff, "Cushing's Syndrome: Important Issues in Diagnosis and Management," The Journal of Clinical Endocrinology \& Metabolism, Vol. 91, No. 10, 2006, pp. 3746-3753. doi:10.1210/jc.2006-0997

[5] J. W. Findling and H. Raff, "Screening and Diagnosis of Cushing's Syndrome," Endocrinology and Metabolism Clinics of North America, Vol. 34, No. 2, 2005, pp. 385402.

[6] M. Castro, P. C. Elias and A. Quidute, "Out-Patient Screening for Cushing's Syndrome: The Sensitivity of the Combination of Circadian Rhytm and Overnight Dexamethasone Suppression Salivary Cortisol Tests," The Journal of Clinical Endocrinology \& Metabolism, Vol. 84, No. 3, 1999, pp. 878-882.

[7] N. Sonino, M. Boscaro, F. Fallo and G. A. Fava, “A Clinical İndex for Rating Severity in Cushing's Syndrome," Psychother, Vol. 69, No. 4, 2000, pp. 216-220.

[8] E. J. Ross and C. Linch, "Cushing's Syndrome-Killing Disease: Discriminatory Value of Signs and Symptoms Aiding Early Diagnosis," The Lancet, Vol. 18, No. 9, 1982, pp. 646-649. 